\title{
Utilitarianism without individual utilities
}

\author{
Thierry Marchant
}

the date of receipt and acceptance should be inserted later

\begin{abstract}
We characterize anonymous utilitarianism in a multi-profile and purely ordinal framework, i.e. without assuming that utilities have been measured beforehand.
\end{abstract}

Keywords utilitarianism · scoring rule

JEL D60 - D71

\section{Introduction}

Many papers on cardinal Social Choice Theory begin with the assumption that individual utilities have been measured by some means, and that we want to aggregate them into a collective preference represented by a Social Welfare Functional $W$. Some axioms like neutrality, anonymity and Pareto are then imposed. These are usually easy to interpret. But in order to arrive at a characterization, more conditions are needed. That is why, in many papers, some invariance conditions are used [e.g. Sen, 1970, d'Aspremont and Gevers, 1977, Roberts, 1980, Bossert, 1991]. These conditions are supposed to represent the informational basis of the social preferences. Contrary to the other conditions, the invariance conditions are not easily interpretable and, often, misunderstood. The reason is that they fail to distinguish between a transformation of the well-being and a transformation of the numerical representation thereof. This problem has been discussed at length in Morreau and Weymark [2016]. Similar discussions, in different contexts, can be found in [Roemer, 1996, Sec. 2.5] and [Marchant, 2008].

To avoid the above-mentioned ambiguity, Morreau and Weymark [2016] introduce a new formalism making explicit reference to the measurement scale being used. This way, they can make a distinction between two kind of invariance conditions: those corresponding to a transformation of the well-being

Ghent University, Ghent 9000, Belgium. E-mail: thierry.marchant@ugent.be 
(without any change of the measurement scale) and those corresponding to a change of the measurement scale (without any change of the well-being). Although this formalism is definitely precise and unambiguous, we think another approach can be enlightening. Instead of supposing that individual utilities have been measured before we consider their aggregation, we suggest to characterize the whole process of measuring individual utilities (by observing individual preferences) and aggregating them. The advantage of this approach is that the statements of characterization theorems are then exempt of any reference to individual utilities. Since individual utilities are not empirically observable, we deem it preferable to avoid using them as primitives of our theories, so as to obtain theorems that are easier to interpret. In doing this, we follow a long tradition: for instance [Arrow, 1963, p.109, bottom] and [Dhillon and Mertens, 1999, p.473, top].

Characterizations of cardinal aggregation procedures without reference to individual utilities can be found in the literature. For instance, Harsanyi's Theorem [Harsanyi, 1955]. This single-profile result has often been criticized for not answering the question it aims to answer [e.g. Weymark, 1991].

More recently, there have been a couple of papers about relative utilitarianism in a purely ordinal setting. Relative utilitarianism is a particular case of utilitarianism in which individual utilities are normalized between 0 and 1 . Dhillon $[1998]^{1}$ characterizes relative utilitarianism using inter alia a strong Pareto condition. The latter is very unusual in the sense that, for each bipartition of the set of voters, it assumes the existence of two social welfare functions satisfying some kind of Pareto condition. A remarkable paper by Dhillon and Mertens [1999] also characterizes relative utilitarianism.

Another paper about relative utilitarianism in a purely ordinal setting is Börgers and Choo [2017b]. Their goal is to represent existing preferences of the individuals and of the social planner. They assume they can observe sufficiently many choices of a social planner to infer his/her preferences.

Let us also mention a paper by Sprumont [2013] about cardinal social choice theory in a purely ordinal framework. He characterizes relative egalitarianism, i.e. an aggregation procedure that ranks alternatives by applying the leximin ordering to the profile of $0-1$ normalized utilities corresponding to the profile of preferences.

In summary, there are few results in the axiomatic literature about cardinal aggregation procedures in a purely ordinal and multi-profile setting: Dhillon [1998], Dhillon and Mertens [1999], Börgers and Choo [2017b], Sprumont [2013]. None of them characterizes the general form of utilitarianism (they focus on relative utilitarianism). The aim of this paper is precisely to present such a result.

To some extent, Smith's characterization of scoring rules [Smith, 1973] could be considered as a characterization of utilitarianism, where scores play the role of individual utilities. The problem with this interpretation of Smith's

1 Börgers and Choo [2017a] have shown that the proof of the main result in Dhillon [1998] is incorrect. Yet, the main result might be correct. 
result is that the choice of a particular score vector is completely arbitrary and not justified by any theory. Our approach is related to Smith's or, more precisely to Young's characterization of scoring rules [Young, 1975] because we consider social choice correspondences and not social welfare functions. Besides, we use a slightly different framework and add some conditions to his so that the use of VNM utilities is completely justified. The reason why we consider social choice correspondences and not social welfare functions is that we use a result by Pivato [2014] which is stated in terms of a social choice correspondence. We are not aware of an equivalent result for social welfare functions.

In the sequel, we consider the following problem. A social planner wants to choose a subset of alternatives from some finite set $X$, taking preferences of voters into account. The social planner wants the choice to possibly depend on individual strengths of preferences or some kind of cardinal information. The voters therefore express their preferences by means of Von NeumannMorgenstern preference relations defined on the set of all lotteries with prizes in $X$. The social planner then uses a social choice correspondence associating a subset of $X$ to each profile of VNM preference relations over lotteries. In Section 2, we characterize the family of all anonymous utilitarian social choice correspondences, i.e. choice sets containing all alternatives for which the (unweighted) sum of individual VNM utilities is maximal. In section 3, we consider a special member of this family: relative utilitarianism. The fourth section is devoted to the logical independence of our axioms and the last section to some open problems or directions for future research.

\section{Characterization of anonymous utilitarianism}

\subsection{Notation and definitions}

Let $X$ be the finite set of alternatives (with $\# X \geq 3$ and typical elements $x, y, z)$ and $\Pi=\{p, q, r, \ldots\}$ be the set of all probability distributions on $X$. Each such probability distribution is called a lottery. Given the lottery $p$ in $\Pi$, the probability that $x$ obtains is denoted by $p_{x} \cdot{ }^{2}$ The lottery such that $x$ obtains with certainty is denoted by $\bar{x}$. It is called a safe lottery. The set of all binary relations on $\Pi$ is $\mathcal{R}=2^{\Pi \times \Pi}$. If $R \in \mathcal{R}$, then $P$ and $I$ respectively denote the asymmetric and the symmetric part thereof. A binary relation $R$ on $\Pi$ is a von Neumann-Morgenstern (VNM) relation [Jensen, 1967] if it satisfies

- weak order: it is transitive, reflexive and complete;

- independence: if $p P q$, then $\lambda p+(1-\lambda) r P \lambda q+(1-\lambda) r$ for all $\lambda \in] 0,1[$;

- continuity: if $p P q$ and $q P r$, then there are $\left.\lambda, \lambda^{\prime} \in\right] 0,1[$ such that $\lambda p+(1-\lambda) r P q$ and $q P \lambda^{\prime} p+\left(1-\lambda^{\prime}\right) r$.

\footnotetext{
2 An anonymous reviewer suggested an alternative interpretation for the lottery $p$ : given a finite time horizon, $p_{x}$ is the proportion of time spent in state $x$ or proportion of time spent consuming good $x$, or ... All models and axioms presented in the sequel can be interpreted with risk or time in mind.
} 
Most other definitions of VNM relations would work equally well. Let $V \subset \mathcal{R}$ be the set of all VNM relations on $\Pi$. We say a binary relation $R$ has an expected utility representation if there exists a mapping $u: X \rightarrow \mathbb{R}$ such that

$$
p R q \Longleftrightarrow \sum_{x \in X} p_{x} u(x) \geq \sum_{x \in X} q_{x} u(x), \text { for all } p, q \in \Pi \text {. }
$$

A binary relation has an expected utility representation as in (1) if and only if it is a VNM relation [Jensen, 1967]. The utility function $u$ in (1) is a VNM utility function; it is unique up to a positive affine transformation.

Given a finite set of agents $N \subset \mathbb{N}$, a profile $\succsim=\left(\succsim_{i}\right)_{i \in N}$ is an element of $V^{N}$ indexed by the elements of $N$, where $\succsim_{i}$ is the preference relation of individual $i$. Let $\mathcal{P}_{N}$ be the set of all possible profiles given $X$ and $N$ and

$$
\mathcal{P}=\bigcup_{\substack{N \subset \mathbb{N} \\ N \text { finite }}} \mathcal{P}_{N}
$$

We define a VNM Social Choice Correspondence (SCC) as a mapping $f: \mathcal{P} \rightarrow$ $2^{X} \backslash \emptyset$, that is, a mapping from the set of all possible profiles to the set of all non-empty subsets of $X$. Notice that the choice set is a subset of $X$ and not of $\Pi$. We want to choose alternatives, i.e. elements of $X$, even though the preferential information we use is defined on the richer set $\Pi$.

Let $\sigma_{X}$ be a permutation on $X$ and $\Sigma$ the set of all such permutations. Then $\sigma_{\Pi}$ is a permutation on $\Pi$ defined by $\left(\sigma_{\Pi}(p)\right)_{x}=p_{\sigma(x)}$ for all $x \in X$ and $p \in \Pi$. Similarly, $\sigma_{\mathcal{R}}$ is a permutation on $\mathcal{R}$ defined by $\sigma_{\Pi}(p) \sigma_{\mathcal{R}}(R) \sigma_{\Pi}(q)$ iff $p R q$ for all $p, q \in \Pi$ and all $R \in \mathcal{R}$. And $\sigma_{\mathcal{P}}$ is a permutation on $\mathcal{P}$ defined by $\sigma_{\mathcal{P}}\left(\left(\succsim_{i}\right)_{i \in N}\right)=\left(\sigma_{\mathcal{R}}\left(\succsim_{i}\right)\right)_{i \in N}$ for all $\left(\succsim_{i}\right)_{i \in N} \in \mathcal{P}$. We henceforth abuse notation and write $\sigma$ without subscript for all these permutations.

A VNM Social Choice Correspondence is anonymous and utilitarian iff it is defined by

$$
f\left(\left(\succsim_{i}\right)_{i \in N}\right)=\underset{x \in X}{\operatorname{argmax}} \sum_{i \in N} u\left(\succsim_{i}, x\right)
$$

where $u: V \times X \rightarrow \mathbb{R}$ is such that

(i) $u(R, \cdot)$ is a VNM utility function, for any $R \in V$,

(ii) $u$ is neutral, i.e., $u(\sigma(R), x)=u(R, \sigma(x))$, for any $R \in V$ and $x \in X$.

The aim of this section is to characterize the family of all anonymous utilitarian VNM Social Choice Correspondences.

To help the reader understand the concept of a neutral VNM utility function, we provide an example of a VNM utility function that violates neutrality. Suppose $X=\{x, y, z\}$. Let $R$ and $R^{\prime}$ be VNM preference relations such that $\bar{y} I 0.4 \bar{x}+0.6 \bar{z}$ and $\bar{x} I^{\prime} 0.4 \bar{y}+0.6 \bar{z}$. Notice that $R^{\prime}$ is obtained from $R$ by permuting $x$ and $y$. So, $R^{\prime}=\sigma(R)$ with $\sigma$ defined by $\sigma(x)=y, \sigma(y)=x$ and $\sigma(z)=z$. Consider a VNM utility function $u$ such that $u(R, x)=1$, $u(R, y)=0.4, u(R, z)=0, u\left(R^{\prime}, y\right)=2, u\left(R^{\prime}, x\right)=0.8, u\left(R^{\prime}, z\right)=0$. This utility function is not neutral because $u(\sigma(R), x)=u\left(R^{\prime}, x\right)=0.8 \neq u(R, \sigma(x))=$ $u(R, y)=0.4$. 
Note that $u$ is not fixed in (2). Yet, if we apply a transformation to $u$, we must respect (i) and (ii). We can distinguish two classes of transformation. First, we can apply a positive affine transformation to $u$, i.e., $u^{\prime}=\alpha u+\beta$, with $\alpha>0$. This results in exactly the same VNM SCC. Second, we can apply a positive affine transformation to $u(\sigma(R), \cdot)$ for some $R \in V$ and for all $\sigma \in \Sigma$, i.e., $u^{\prime}(\sigma(R), \cdot)=\alpha u(\sigma(R), \cdot)+\beta$. This yields a different VNM SCC whenever $\alpha \neq 1$. Hence, (2) does not define a single VNM SCC but a family.

We can for instance choose $u$ so that $u(R, \cdot)$ is normalized (except if $R$ is the trivial preference relation ${ }^{3}$ ), that is, $u(R, x)=1$ if $\bar{x} R \bar{z}$ for all $z \in X$ and $u(R, y)=0$ if $\bar{z} R \bar{y}$ for all $z \in X$. It is easy to check that $u$ is neutral. The corresponding VNM SCC is then equivalent to relative utilitarianism [Dhillon and Mertens, 1999]. We can also choose $u$ so that the range of $u(R, \cdot)$ goes from zero to the number of equivalence classes of safe lotteries in $R$. Many other choices are of course possible.

\subsection{Standard axioms}

In order to characterize the family of all anonymous utilitarian VNM SCCs, we use a result by Pivato [2014] extending a result of Myerson [1995], which is itself an extension of a result of Young $[1975]^{4}$. Our axioms are therefore very similar to those of Young [1975]. We present them hereunder without much comment, because they have been extensively discussed elsewhere. The first condition says that all alternatives are treated equally.

A 1 Neutrality. For each profile $\succsim \in \mathcal{P}$ and permutation $\sigma$ on $X$,

$$
\sigma(f(\succsim))=f(\sigma(\succsim)) \text {. }
$$

The second condition says that all agents are treated equally.

A 2 Anonymity. For all finite $N \subset \mathbb{N}$, all profiles $\succsim, \succsim^{\prime} \in \mathcal{P}_{N}$ and every permutation $\gamma$ on $N$ such that $\succsim_{i}=\succsim_{\gamma(i)}^{\prime}$, for all $i \in N$,

$$
f(\succsim)=f\left(\succsim^{\prime}\right)
$$

Young [1975] groups these two conditions under the name 'Symmetry'.

We introduce a new piece of notation before next condition. Let $\succsim=\left(\succsim_{i}\right.$ )$_{i \in N}$ and $\succsim^{\prime}=\left(\succsim_{i}\right)_{i \in M}$ be two profiles with $N \cap M=\emptyset$. Then $\succsim^{\prime \prime}=\succsim \circ \succsim^{\prime}$ is the profile in $\mathcal{P}_{N \cup M}$ defined by

$$
\succsim_{i}^{\prime \prime}=\left\{\begin{array}{l}
\succsim_{i} \text { if } i \in N \\
\succsim_{i}^{\prime} \text { if } i \in M .
\end{array}\right.
$$

If $f(\succsim)$ is the choice set of an agent group $N$ and $f\left(\succsim^{\prime}\right)$ is the choice set of another agent group $M$ disjoint from $N$, and if $f\left(\succsim^{\prime}\right) \cap f\left(\succsim^{\prime}\right) \neq \emptyset$, then the group $N \cup M$ should choose precisely the alternatives in $f(\succsim) \cap f\left(\succsim^{\prime}\right)$. Formally,

\footnotetext{
3 The preference relation $R$ is trivial if $\bar{x} R \bar{y}$ for all $x, y \in X$.

4 The latter is closely linked to [Smith, 1973]
} 
A 3 Separability. Let $\succsim=\left(\succsim_{i}\right)_{i \in N}$ and $\succsim^{\prime}=\left(\succsim_{i}\right)_{i \in M}$ be two profiles with $N \cap M=\emptyset$. If $f(\succsim) \cap f\left(\succsim^{\prime}\right) \neq \emptyset$, then $f\left(\succsim^{\prime} \succsim^{\prime}\right)=f(\succsim) \cap f\left(\succsim^{\prime}\right)$.

This is what Young [1975] calls Consistency while Myerson [1995] calls it Reinforcement. We call it Separability, like Smith [1973].

Let $\succsim=\left(\succsim_{i}\right)_{i \in N}$ and $\succsim^{\prime}=\left(\succsim_{i}^{\prime}\right)_{i \in M}$ be two profiles. We say $\succsim$ and $\succsim^{\prime}$ are isomorphic if there is a bijection $\mu: N \rightarrow M$ such that $\succsim_{i}=\succsim_{\mu(i)}^{\prime}$ for all $i \in N$. If $f\left(\succsim^{1}\right)$ is the choice set of a certain group $N^{1}$, then given any second group $M$ disjoint from $N^{1}$ and with preference profile $\succsim^{\prime}$, we can replicate the first group and its preference profile (using isomorphic profiles) a sufficient number of times so that it overwhelms the second group in a combined profile and yield a subset of $f\left(\succsim^{1}\right)$ as choice set. This kind of continuity requirement is our Archimedean condition.

A 4 Archimedeanness. Let $\left\{N^{j}\right\}_{j \in \mathbb{N}}$ be a collection of disjoint subsets of $\mathbb{N}$, all of size $n$. Suppose $\left\{\succsim^{j}\right\}_{j \in \mathbb{N}}$ is a collection of isomorphic profiles in $\mathcal{P}_{N^{j}}$ and $\succsim^{\prime} \in \mathcal{P}_{M}$ with $\left(\bigcup_{j \in \mathbb{N}} N^{j}\right) \cap M=\emptyset$. Then there exists $h \in \mathbb{N}$ such that, for every $k>h$,

$$
f\left(\succsim^{1} \circ \ldots \circ \succsim^{k} \circ \succsim^{\prime}\right) \subseteq f\left(\succsim^{1}\right) .
$$

This is exactly Myerson's (1995) Overwhelming Majority. The next condition is a standard Pareto condition [e.g. Donaldson and Weymark, 1988].

A 5 Weak Pareto. For any profile $\succsim=\left(\succsim_{i}\right)_{i \in N} \in \mathcal{P}$, if there are $x, y \in X$ such that $\bar{x} \succ_{i} \bar{y}$ for all $i \in N$, then $y \notin f(\succsim)$.

In our first result, we use a weakening o fthis condition and a strict version thereof. Let $(R)_{i}$ denote a profile with a single voter $i$ and a single preference relation $R$.

A 6 Non-Triviality. There exists $R \in V$ such that $f\left((R)_{i}\right) \neq X$.

A 7 Strict Pareto. For any profile $\succsim=\left(\succsim_{i}\right)_{i \in N} \in \mathcal{P}$, if there are $x, y \in X$ such that $\bar{x} \succsim_{i} \bar{y}$ for all $i \in N$ and $\bar{x} \succ_{j} \bar{y}$ for some $j \in N$, then $y \notin f(\succsim)$.

\subsection{Preliminary result}

Using the conditions of previous section, we state a preliminary result that can almost be considered as a corollary to a result by Pivato [2014].

Proposition 1 Let \#X $\geq 3$. A VNM SCC f satisfies Neutrality, Anonymity, Separability, Archimedeanness and Non-Triviality iff there exists $u: V \times X \rightarrow$ $\mathbb{R}$ such that

$$
f\left(\left(\succsim_{i}\right)_{i \in N}\right)=\underset{x \in X}{\operatorname{argmax}} \sum_{i \in N} u\left(\succsim_{i}, x\right) .
$$

with $u$ neutral, i.e., $u(\sigma(R), x)=u(R, \sigma(x))$, for any $R \in V, \sigma \in \Sigma$ and $x \in X$. If $f$ also satisfies Strict Pareto, then $u(R, x) \geq u(R, y) \Longleftrightarrow \bar{x} R \bar{y}$, for all $x, y \in X$ and all $R \in V$. 
Let us notice that, without Strict Pareto, the mapping $u$ is not necessarily a VNM utility function and not even a utility function. It can be anything, provided it is not constant (so as to satisfy Non-Triviality) and neutral. Yet, with Strict Pareto, the mapping $u$ is a utility function and Proposition 1 can thus be considered as a first characterization of utilitarianism in a very general sense, leaving full freedom for the choice of $u$ as long as $u(R, \cdot)$ is a utility representation of $R$.

It is also important to notice that using a VNM SCC $f$ defined by (3) already implies some interpersonal comparisons. Indeed, if two voters $i, j$ have the same VNM preference relation $R$, then the difference $u(R, x)-u(R, y)$ is the same number for both of them, although one could argue that the difference in well-being is not necessarily the same for $i$ and $j$. Consequently, the choice set is the same for the profile where voter $i$ is replaced by voter $j$. Moreover, if two voters have preferences $R$ and $R^{\prime}$, such that $R^{\prime}=\sigma(R)$ for some $\sigma \in \Sigma$, then the corresponding mappings $u(R, \cdot)$ and $u\left(R^{\prime}, \cdot\right)$ are identical up to a permutation. So, if we have a profile with only two voters, with preferences $R$ and $R^{\prime}$ as above, and if $\sigma(x)=y$ and $\sigma(y)=x$, then both $x$ and $y$ belong to $f\left(R, R^{\prime}\right)$ or none of them does.

Proof of Proposition 1. Let us assume $f$ satisfies all five axioms. Let $\delta$ : $\mathcal{P} \times V \rightarrow \mathbb{N}$ be a mapping such that $\delta(\succsim, R)$ is the number of individuals having the preference $R$ in the profile $\succsim$. Let $\succsim \in \mathcal{P}_{N}$ and $\succsim^{\prime} \in \mathcal{P}_{N^{\prime}}$ be such that $\delta(\succsim, R)=\delta\left(\succsim^{\prime}, R\right)$ for all $R \in V$. Let $(R)_{i \in M}$ denote the profile such that each agent in $M$ has the same preferences $R$. By Neutrality, $f\left(\left(\Pi^{2}\right)_{i \in N^{\prime} \backslash N}\right)=$ $f\left(\left(\Pi^{2}\right)_{i \in N \backslash N^{\prime}}\right)=X$. By Separability, $f\left(\succsim \circ\left(\Pi^{2}\right)_{i \in N^{\prime} \backslash N}\right)=f(\succsim)$ and $f\left(\succsim^{\prime}\right.$ $\left.\circ\left(\Pi^{2}\right)_{i \in N \backslash N^{\prime}}\right)=f\left(\succsim^{\prime}\right)$. Since $\succsim \circ\left(\Pi^{2}\right)_{i \in N^{\prime} \backslash N}$ and $\succsim^{\prime} \circ\left(\Pi^{2}\right)_{i \in N \backslash N^{\prime}}$ both belong to $\mathcal{P}_{N \cup N^{\prime}}$ and $\delta\left(\succsim \circ\left(\Pi^{2}\right)_{i \in N^{\prime} \backslash N}, R\right)=\delta\left(\succsim^{\prime} \circ\left(\Pi^{2}\right)_{i \in N \backslash N^{\prime}}, R\right)$ for all $R \in V$, Anonymity implies $f(\succsim)=f\left(\succsim^{\prime}\right)$. Hence $f(\succsim)$ depends only on $(\delta(\succsim, R))_{R \in V}$. In other words, there exists $F: \mathbb{N}^{V} \rightarrow 2^{X} \backslash \emptyset$ such that $F\left((\delta(\succsim, R))_{R \in V}\right)=$ $f(\succsim)$ for all $\succsim \in \mathcal{P}$. Because $f$ satisfies Neutrality, Non-Triviality, Separability and Archimedeanness, $F$ satisfies Neutrality, Non-Triviality, Reinforcement and Overwhelming Majority as defined in Pivato [2014]. By Proposition A.1 in Pivato [2014], there exists $u: V \times X \rightarrow \mathbb{R}$ such that

$$
F\left((\delta(\succsim, R))_{R \in V}\right)=\underset{x \in X}{\operatorname{argmax}} \sum_{R \in V} \delta(\succsim, R) u(R, x)
$$

with $u$ neutral, i.e., $u(\sigma(R), x)=u(R, \sigma(x))$, for any $R \in V, \sigma \in \Sigma$ and $x \in X$. Clearly, Equation (4) can be rewritten in terms of $f$ as (3).

Let us now suppose $f$ also satisfies Strict Pareto. Let $R$ be a relation such that $\bar{x} I \bar{y}$. Then, since $u$ is neutral, it readily follows that $u(R, x)=u(R, y)$. Let now $R$ be a relation such that $\bar{x} P \bar{w}$ for all $w \in X$. By Strict Pareto, $\{x\}$ is the choice set of the profile containing only $R$. Hence, $u(R, x)>$ $u(R, w)$ for all $w \in X$. Let finally $R$ be a relation such that $\bar{x} P \bar{y}$ and suppose for contradiction $u(R, x) \leq u(R, y)$. Consider a relation $R^{\prime} \in V$ such that $\bar{x} I^{\prime} \bar{y}, \bar{x} P^{\prime} \bar{w}$ and $\bar{w} I^{\prime} \bar{z}$ for all $z \in X \backslash\{x, y, w\}$. We have $u\left(R^{\prime}, x\right)=u\left(R^{\prime}, y\right)>u\left(R^{\prime}, w\right)=u\left(R^{\prime}, z\right)$ for all $z \in X \backslash\{x, y, z\}$. Let 
$N^{1} \subset \mathbb{N}$ be such that $j \notin N^{1}$ and let $\succsim=\left((R)_{j} \circ\left(R^{\prime}\right)_{i \in N^{1}}\right)$. For arbitrary large $N^{1}$, we have $u(R, y)+\sum_{i \in N^{1}} u\left(R^{\prime}, y\right) \geq u(R, x)+\sum_{i \in N^{1}} u\left(R^{\prime}, x\right)>$ $u(R, z)+\sum_{i \in N^{1}} u\left(R^{\prime}, z\right)$ for all $z \in X \backslash\{x, y\}$. Hence $y \in f(\succsim)$ but Strict Pareto implies $y \notin f(\succsim)$. This contradiction proves that $u(R, x)>u(R, y)$. In summary, $u(R, x) \geq u(R, y) \Longleftrightarrow \bar{x} R \bar{y}$, for all $x, y \in X$ and all $R \in V$.

The reader may have noticed that the proof does not rely on the fact that a profile consists of VNM relations. The same theorem would therefore hold with any set of binary relations as long as the set is closed under permutations of $X$.

\subsection{A new condition}

All axioms presented so far are standard in the social choice literature. Usually, they are imposed on SCCs acting on profiles of preference relations on unstructured sets, but nothing prevents us from imposing them on a SCC acting on profiles of preference relations defined on a structured set (e.g., $\Pi$ ), as we just did. Yet, none of these axioms makes use of the structure of $\Pi$; none of them helps us to exploit the potentially cardinal information contained in the VNM preference relations. Our last condition precisely does this.

A quick look at (2) shows that not only the number of agents preferring $x$ over $y$ (resp. $y$ over $x$ ) matters when comparing $x$ and $y$, but also the extent by which they prefer $x$ over $y$, i.e. the difference $u\left(\succsim_{i}, x\right)-u\left(\succsim_{i}, y\right)$. Hence, many agents slightly preferring $x$ over $y$ can be overruled by few agents strongly preferring $y$ over $x$. In other words, there is some kind of interplay between the number of agents preferring $x$ over $y$ and the extent by which they prefer $x$ over $y$. Let us formalize this without making reference to the utilities, but only to the primitives.

Suppose a group of $n^{1}$ agents all have the same preferences: they strictly prefer $x$ to $y$ to $z$. The other alternatives have any other position. Moreover, all agents in this group are indifferent between the safe lottery $\bar{y}$ and the mixture $\lambda \bar{x}+(1-\lambda) \bar{z}$, with $\lambda=0.9$. We might consider this as relevant information about the relative standing of $y$ vis-à-vis $x$ and $z$. More specifically, we might consider that they support $x$ more than $y$, but only slightly, while they strongly support $x$ against $z$. Suppose another group of $n^{2}$ agents have the same preferences as the $n^{1}$ agents, up to a permutation $\sigma$ such that $\sigma(x)=y$, $\sigma(z)=x$. Hence, all agents in the second group prefer $y$ to $x$ and they support $y$ against $x$ to the same extent as agents in the first group support $x$ to $z$, that is strongly. So, the ratio "support for $x$ against $y$ in group 1 over support for $y$ against $x$ in group 2 " is $(1-\lambda) / 1$. Suppose we consider a society consisting only of these two groups of agents. If we want to account for the strength or intensity of preferences, and if we accept the idea that many agents supporting $x$ slightly more than $y$ can be compensated by few agents supporting $y$ much more than $x$, then we may be tempted to consider that this compensation occurs when $n^{2} / n^{1}=1-\lambda$. In that case, $x$ is chosen if and only if $y$ is chosen. 
The formal statement of this condition is more complex: we do not impose this condition for all groups of agents, but only for all groups included in some infinite subset of $\mathbb{N}$ because we do not want to exclude the possibility that some agents have a different status and are treated differently. Let $(R)_{i \in N}$ denote a profile consisting of $\# N$ copies of the preference relation $R$.

A 8 VNM-Comparability. There exists an infinite subset $O$ of $\mathbb{N}$ such that, whenever

- $R \in V$ is such that $\bar{x} P \bar{y} P \bar{z}$ and $\bar{y} I \lambda \bar{x}+(1-\lambda) \bar{z}$,

- $N^{1}$ and $N^{2}$ are disjoint subsets of $O$,

$-\sigma \in \Sigma$ is such that $\sigma(x)=y, \sigma(z)=x$

- \# $N^{2} / \# N^{1}=1-\lambda$,

then

$$
x \in f\left((R)_{i \in N^{1}} \circ(\sigma(R))_{i \in N^{2}}\right) \Longleftrightarrow y \in f\left((R)_{i \in N^{1}} \circ(\sigma(R))_{i \in N^{2}}\right) .
$$

As far as we know, this condition has never been discussed in the literature. We do not claim that it is compelling or even appealing. Paraphrasing Sen [1976], p.254, it is not designed "to provide an axiomatic justification of" utilitarianism. Instead, we chose "a set of axioms with the focus on transparency rather than on immediate appeal" [Sen, 1976, p.259]. ${ }^{5}$ For a discussion of whether risk preferences may inform social choices over non risky options, see [e.g. Chambers and Echenique, 2012, Fleurbaey and Zuber, 2017].

The interest of our VNM-Comparability is that it allows us to characterize the anonymous utilitarian VNM SCC exclusively in terms of empirically observable primitives, thereby escaping the ambiguities of the social welfare functionals approach, as discussed by Morreau and Weymark [2016].

\subsection{Main result}

We are now ready to state our main characterization theorem.

Theorem 1 Let $\# X \geq 3$. A VNM SCC satisfies Neutrality, Anonymity, Separability, Archimedeanness, Weak Pareto and VNM-Comparability iff it is anonymous and utilitarian.

This result is not a justification of anonymous utilitarianism, because, as mentioned earlier, we do not consider our axioms as compelling. In some contexts, a social planner might consider them as appealing or reasonable and therefore decide to use an anonymous utilitarian SCC. In other contexts, another social planner might have strong arguments against our axioms and thus decide not to use an anonymous utilitarian SCC. In both cases, our axioms constitute unambiguous elements that can be used in a debate about the anonymous utilitarian VNM SCC.

\footnotetext{
5 Our view of the axiomatic analysis is also close in spirit to that of Thomson [2001], in a different domain.
} 
Some kind of interpersonal comparability was already implied by Proposition 1. The addition of VNM Comparability in Theorem 1, for characterizing anonymous utilitarianism, makes clear how some kind of cardinal information, latent in the individual preference relations, is used to make interpersonal comparisons of differences of utilities. But, unlike invariance conditions such as, e.g. Cardinal Unit Comparability [Roberts, 1980], it does this without ever mentioning individual utilities.

Proof of Theorem 1. Let us assume $f$ satisfies all six axioms. Weak Pareto implies Non-Triviality and, thanks to Proposition $1, f$ is defined by (3). We must now prove that $u(R, \cdot)$ is a VNM utility function representing $R$, for any $R \in V$. To this end, we consider three exhaustive cases.

1. $R$ has only one equivalence class. Formally, $R \in V$ is such that $\bar{x} I \bar{y}$, for all $x, y \in X$. Since $u$ is neutral, $u(R, x)=u(R, y)$ for all $x, y \in X$ and $u(R, \cdot)$ is therefore a VNM utility function representing $R$.

2. $R$ has more than one equivalence class, but all safe lotteries are grouped in exactly two equivalence classes. Formally, $R \in V$ is such that $\bar{x} P \bar{y}$ and $[\bar{z} I \bar{x}$ or $\bar{z} I \bar{y}]$, for all $z \in X$. Define $A=\{z \in X: \bar{z} I \bar{x}\}$. By Weak Pareto, $f(R) \cap(X \backslash A)=\emptyset$. Since $f(R) \neq \emptyset$, there is $w \in A$ such that $w \in f(R)$. By Neutrality, $f(R)=A$ and $u(R, z)=u(R, x)$ for all $z \in A$. Since $u$ is neutral, $u(R, z)=u(R, y)$ for all $z \notin A$. Since $x \in f(R)$ and $y \notin f(R)$, we have $u(R, x)>u(R, y)$. In conclusion, $u(R, \cdot)$ is a VNM utility function representing $R$.

3. $R$ has more than one equivalence class and the safe lotteries are grouped in $k$ equivalence classes, with $k \geq 3$. Let us rename the alternatives as $x_{1}, \ldots, x_{k}, \ldots, x_{\# X}$ so that $x_{1} P x_{2} P \ldots P x_{k}$. We build $u(R, \cdot)$ in a number of steps.

(a) Utility of $x_{1}$ and $x_{k}$. Choose any arbitrary value for $x_{1}$ and $x_{k}$, provided $x_{1}>x_{k}$.

(b) Utility of $x_{2}$. Let $\lambda_{2}$ be such that $\bar{x}_{2} I \lambda_{2} \bar{x}_{1}+\left(1-\lambda_{2}\right) \bar{x}_{k}$ and assume for now $\lambda_{2} \in \mathbb{Q}$. Let $N^{1}, N^{2} \subset O$ be such that $\# N^{2} / \# N^{1}=1-\lambda_{2}$. This is possible because $\lambda_{2} \in \mathbb{Q}$. Let $\sigma_{2}$ be such that $\sigma_{2}\left(x_{k}\right)=x_{1}$ and $\sigma_{2}\left(x_{i}\right)=x_{i+1}$ for all $i \in\{1, \ldots k-1\}$. Notice that this permutation is like $\sigma$ in the statement of VNM-Comparability. By Weak Pareto, $\left\{x_{3}, \ldots, x_{k}\right\} \cap f\left((R)_{i \in N^{1}} \circ\left(\sigma_{2}(R)\right)_{i \in N^{2}}\right)=\emptyset$. Hence $x_{1}$ or $x_{2}$ belongs to $f\left((R)_{i \in N^{1}} \circ\left(\sigma_{2}(R)\right)_{i \in N^{2}}\right)$. By VNM-Comparability, they both belong to the choice set. By virtue of $(3)$, this implies

$$
\sum_{i \in N^{1}} u\left(R, x_{1}\right)+\sum_{i \in N^{2}} u\left(\sigma_{2}(R), x_{1}\right)=\sum_{i \in N^{1}} u\left(R, x_{2}\right)+\sum_{i \in N^{2}} u\left(\sigma_{2}(R), x_{2}\right) .
$$

Since $u$ is neutral and $\sigma_{2}\left(x_{k}\right)=x_{1}$ and $\sigma_{2}\left(x_{1}\right)=x_{2}$,

$$
\sum_{i \in N^{1}} u\left(R, x_{1}\right)+\sum_{i \in N^{2}} u\left(R, x_{k}\right)=\sum_{i \in N^{1}} u\left(R, x_{2}\right)+\sum_{i \in N^{2}} u\left(R, x_{1}\right) .
$$


Therefore

$$
\# N^{1} u\left(R, x_{1}\right)+\# N^{2} u\left(R, x_{k}\right)=\# N^{1} u\left(R, x_{2}\right)+\# N^{2} u\left(R, x_{1}\right)
$$

or

$$
\frac{u\left(R, x_{1}\right)-u\left(R, x_{2}\right)}{u\left(R, x_{1}\right)-u\left(R, x_{k}\right)}=\frac{\# N^{2}}{\# N^{1}}=1-\lambda_{2}
$$

or

$$
\frac{u\left(R, x_{2}\right)-u\left(R, x_{k}\right)}{u\left(R, x_{1}\right)-u\left(R, x_{k}\right)}=\lambda_{2} .
$$

This proves that, when $\lambda_{2} \in \mathbb{Q}, u(R, \cdot)$ restricted to $\left\{x_{1}, x_{2}, x_{k}\right\}$ coincides with a VNM utility function. If $\lambda_{2} \notin \mathbb{Q}$, we can "squeeze"

$$
\frac{u\left(R, x_{1}\right)-u\left(R, x_{2}\right)}{u\left(R, x_{1}\right)-u\left(R, x_{k}\right)}
$$

between two sequences of ratios $\# N^{2} / \# N^{1}$ both converging to $1-\lambda_{2}$, respectively from below and from above.

(c) Utility of $x_{i}, i \in\{3, \ldots, k-1\}$. Let $\lambda_{i}$ be such that $\bar{x}_{i} I \lambda_{i} \bar{x}_{1}+(1-$ $\left.\lambda_{i}\right) \bar{x}_{k}$ and assume for now $\left(1-\lambda_{i}\right) /\left(1-\lambda_{2}\right) \in \mathbb{Q}$. Let $N^{1}, N^{2} \subset O$ be such that $\# N^{2} / \# N^{1}=\left(1-\lambda_{i}\right) /\left(1-\lambda_{2}\right)$. This is possible because $\left(1-\lambda_{i}\right) /\left(1-\lambda_{2}\right) \in \mathbb{Q}$. Let $\sigma_{i}$ be such that $\sigma_{i}\left(x_{1}\right)=x_{i}, \sigma_{i}\left(x_{j}\right)=x_{j-1}$ for all $j \in\{2, \ldots, i\}$ and $\sigma_{i}\left(x_{j}\right)=x_{j}$ for all $j \in\{i+1, \ldots k\}$. Notice that $\sigma_{i}(R), R$ and $\sigma_{i}^{-1}$ are respectively like $R, \sigma(R)$ and $\sigma$ in the statement of VNM-comparability. By Weak Pareto, $\left\{x_{2}, \ldots, x_{i-1}, x_{i+1}, \ldots, x_{k}\right\} \cap$ $f\left((R)_{i \in N^{1}} \circ\left(\sigma_{i}(R)\right)_{i \in N^{2}}\right)=\emptyset$. Hence $x_{1}$ or $x_{i}$ belongs to $f\left((R)_{i \in N^{1}} \circ\right.$ $\left.\left(\sigma_{i}(R)\right)_{i \in N^{2}}\right)$. By VNM-Comparability, they both belong to the choice set. By virtue of (3), this implies

$$
\sum_{i \in N^{1}} u\left(R, x_{1}\right)+\sum_{i \in N^{2}} u\left(\sigma_{i}(R), x_{1}\right)=\sum_{i \in N^{1}} u\left(R, x_{i}\right)+\sum_{i \in N^{2}} u\left(\sigma_{i}(R), x_{i}\right) .
$$

Since $u$ is neutral and $\sigma_{i}\left(x_{2}\right)=x_{1}$ and $\sigma_{i}\left(x_{1}\right)=x_{i}$,

$$
\sum_{i \in N^{1}} u\left(R, x_{1}\right)+\sum_{i \in N^{2}} u\left(R, x_{2}\right)=\sum_{i \in N^{1}} u\left(R, x_{i}\right)+\sum_{i \in N^{2}} u\left(R, x_{1}\right) .
$$

Therefore

$$
\# N^{1} u\left(R, x_{1}\right)+\# N^{2} u\left(R, x_{2}\right)=\# N^{1} u\left(R, x_{i}\right)+\# N^{2} u\left(R, x_{1}\right)
$$

or

$$
\frac{u\left(R, x_{1}\right)-u\left(R, x_{i}\right)}{u\left(R, x_{1}\right)-u\left(R, x_{2}\right)}=\frac{\# N^{2}}{\# N^{1}}=\frac{1-\lambda_{i}}{1-\lambda_{2}} .
$$

Let us multiply this equation by (5). We obtain

$$
\frac{u\left(R, x_{1}\right)-u\left(R, x_{i}\right)}{u\left(R, x_{1}\right)-u\left(R, x_{k}\right)}=1-\lambda_{i} .
$$


This proves that, when $\left(1-\lambda_{i}\right) /\left(1-\lambda_{2}\right) \in \mathbb{Q}, u(R, \cdot)$ restricted to $\left\{x_{1}, x_{i}, x_{k}\right\}$ coincides with a VNM utility function. If $\left(1-\lambda_{i}\right) /\left(1-\lambda_{2}\right) \notin$ $\mathbb{Q}$, we can "squeeze"

$$
\frac{u\left(R, x_{1}\right)-u\left(R, x_{i}\right)}{u\left(R, x_{1}\right)-u\left(R, x_{2}\right)}
$$

between two sequences of ratios $\# N^{2} / \# N^{1}$ both converging to $(1-$ $\left.\lambda_{i}\right) /\left(1-\lambda_{2}\right)$, respectively from below and from above.

So, for every $i \in\{2, \ldots, k-1\}, u(R, \cdot)$ restricted to $\left\{x_{1}, x_{i}, x_{k}\right\}$ coincides with a VNM utility function. In summary, $u(R, \cdot)$ is a VNM utility function representing $R$.

So, in any case, $u(R, \cdot)$ is a VNM utility function representing $R$.

\section{A characterization of relative utilitarianism}

There are characterizations of relative utilitarianism in the literature, in a multi-profile setting and without the assumption that utilities have been measured beforehand (see Section 1). It is nevertheless interesting to know what needs to be added to Theorem 1 in order to single out relative utilitarianism. Let us define the relative utilitarian VNM Social Choice Correspondence by

$$
f\left(\left(\succsim_{i}\right)_{i \in N}\right)=\underset{x \in X}{\operatorname{argmax}} \sum_{i \in N} u\left(\succsim_{i}, x\right)
$$

where $u: V \times X \rightarrow \mathbb{R}$ is such that

(a) $u(R, \cdot)$ is a VNM utility function, for any $R \in V$,

(b) $\bar{x} R \bar{y}$ (resp. $\bar{y} R \bar{x}$ ) for all $y \in X$, with at least one strict preference, implies $u(R, x)=1$ (resp. 0).

Notice that this defines a unique VNM SCC. Besides, this VNM SCC is anonymous and utilitarian. Indeed, (a) and (b) together imply that $u$ is neutral. So, the relative utilitarian VNM SCC satisfies all conditions of Theorem 1. The question is: which extra condition is satisfied by the relative utilitarian VNM SCC and only by this one. The answer turns out to be simple: when there are two agents, if a top ranked alternative of the first agent is a bottom ranked alternative of the second agent and vice versa, then both alternatives are chosen or none is chosen.

A 9 Ordinal Comparability. There exists an infinite subset $O$ of $\mathbb{N}$ such that, whenever

$-R \in V$ is not trivial and is such that $\bar{x} R \bar{w} R \bar{y}$ for all $w \in X$,

$-R^{\prime} \in V$ is not trivial and is such that $\bar{y} R^{\prime} \bar{w} R^{\prime} \bar{x}$ for all $w \in X$,

$-i, j \in O, i \neq j, N \subset O$ and $\{i, j\} \cap N=\emptyset$,

then

$$
x \in f\left((R)_{i} \circ\left(R^{\prime}\right)_{j}\right) \Longleftrightarrow y \in f\left((R)_{i} \circ\left(R^{\prime}\right)_{j}\right) .
$$


The structure of this condition is similar to that of VNM-Comparability. We call it Ordinal Comparability because it does not exploit the structure of $\Pi$; it is possible to impose it on a SCC acting on profiles of preference relations defined on abstract unstructured sets.

Theorem 2 A VNM SCC satisfies Neutrality, Anonymity, Separability, Archimedeanness, Weak Pareto, VNM-Comparability and Ordinal Comparability iff it is the relative utilitarian VNM SCC defined by (6).

There are many differences between [Dhillon and Mertens, 1999] and our result. First, our set of voters is variable while theirs is fixed. Second, we characterize a procedure for aggregating preference relations into a choice set while they aggregate preference relations into a social preference relation. We consider these two differences as minor and almost technical. A more fundamental difference is that our choice set never contains a lottery while their social preference relations is defined on the set of all lotteries (and is VNM). In our framework, lotteries play an instrumental role: we are not interested in lotteries, but we use them for obtaining cardinal information about the alternatives. Depending on what we are interested in (lotteries over alternatives of just alternatives), their result or ours may be more relevant.

Proof of Theorem 2. By Theorem 1, $f$ is anonymous and utilitarian. Let $R$ be a non-trivial relation in $V$. We suppose without loss of generality that $\bar{x} R \bar{w} R \bar{y}$ for all $w \in X$. Let $R^{\prime}$ be the relation uniquely defined by $\bar{y} P^{\prime} \bar{x}$ and $\bar{w} I^{\prime} \bar{x}$ for all $w \in X$. Then $R$ and $R^{\prime}$ are as in the statement of Ordinal Comparability. Let $i, j$ and $N \subset O$ be as in the statement of Ordinal Comparability. For every $w \in X \backslash\{x, y\}$, we have $w \notin \in f\left((R)_{i} \circ\left(R^{\prime}\right)_{j}\right)$ because $u(R, x)>$ $u(R, w)$ and $u\left(R^{\prime}, x\right)=u\left(R^{\prime}, w\right)$ so that $u(R, x)+u\left(R^{\prime}, x\right)>u(R, w)+u\left(R^{\prime}, w\right)$. Hence $x$ or $y$ belong to the choice set and, thanks to Ordinal Comparability, both $x$ and $y$ belong to $f\left((R)_{i} \circ\left(R^{\prime}\right)_{j}\right)$. By virtue of $(3)$, this implies $u(R, x)+u\left(R^{\prime}, x\right)=u(R, y)+u\left(R^{\prime}, y\right)$ or $u(R, x)-u(R, y)=u\left(R^{\prime}, y\right)-u\left(R^{\prime}, x\right)$. Since this is true for every relation $R$, the difference between the maximal and minimal utilities is the same for every preference relation. We can suppose without loss of generality that this difference is equal to 1 and that the minimal utility is zero.

At this point, the reader may wonder what we obtain if we impose Ordinal Comparability without VNM-Comparability. It is fairly easy to prove the following result (the proof is left to the reader).

Theorem 3 A VNM SCC satisfies Neutrality, Anonymity, Separability, Archimedeanness, Strict Pareto and Ordinal Comparability iff iff there exists $u: V \times X \rightarrow \mathbb{R}$ such that (3) holds with u neutral, normalized and such that $u(R, \cdot)$ is a utility representation of $R$. 


\section{Logical independence of the conditions}

Examples 1-5 below show that all conditions of Proposition 1 are logically independent. Similarly, Examples $1-6$ show that all conditions of Theorem 3 are logically independent. Unfortunately, we are not able to prove the independence of the conditions in Theorem 1 or 2. Indeed, Archimedeanness might be implied by the other conditions of Theorem 1 but Example 7 nevertheless shows that VNM-Comparability is not implied by the other conditions of Theorem 2. Proving the independence of the conditions (or providing alternative characterizations with weaker conditions) is therefore left as an open problem.

Example 1 (Neutrality) Let $x, y$ be distinct elements of $X$ and let $V^{*}$ be a proper subset of $V$ containing all relations $R \in V$ such that $\bar{x} R \bar{w} R \bar{y}$ or $\bar{y} R \bar{w} R \bar{x}$ for all $w \in X$. Define $g: V \times X \rightarrow \mathbb{R}$ so that, for all $R \in V, g(R, \cdot)$ is the normalized VNM utility function representing $R$ (except for the trivial preference relation). Define $u: V \times X \rightarrow \mathbb{R}$ by

$$
u(R, \cdot)= \begin{cases}g(R, \cdot) & \text { if } R \in V^{*} \\ 2 g(R, \cdot) & \text { otherwise }\end{cases}
$$

and $f$ by

$$
f\left(\left(\succsim_{i}\right)_{i \in N}\right)=\underset{x \in X}{\operatorname{argmax}} \sum_{i \in N} u\left(\succsim_{i}, x\right) .
$$

This VNM SCC obviously violates Neutrality. The reason it satisfies VNMComparability is that the relations $R$ and $\sigma(R)$ in the statement of VNMComparability both belong to $V^{*}$ or both to $V \backslash V^{*}$. The reason it satisfies Ordinal Comparability is that the relations $R$ and $R^{\prime}$ in the statement of Ordinal Comparability both belong to $V^{*}$ or both to $V \backslash V^{*}$. The other conditions are clearly satisfied.

Example 2 (Anonymity) Let $u: V \times X \rightarrow \mathbb{R}$ be such that, for all $R \in V$, $u(R, \cdot)$ is a VNM utility function representing $R$ and $u$ is neutral. Let $O$ be any proper infinite subset of $\mathbb{N}$; for instance the set of all even natural numbers. Define

$$
f\left(\left(\succsim_{i}\right)_{i \in N}\right)=\underset{x \in X}{\operatorname{argmax}}\left(\sum_{i \in N \backslash O} 2 u\left(\succsim_{i}, x\right)+\sum_{i \in N \cap O} u\left(\succsim_{i}, x\right)\right) .
$$

Anonymity is blatantly violated. To understand why $f$ satisfies VNM-Comparability and Ordinal Comparability, notice that both conditions only apply to agents in $O$. In that case, $f$ can be rewritten as

$$
f\left(\left(\succsim_{i}\right)_{i \in N}\right)=\underset{x \in X}{\operatorname{argmax}} \sum_{i \in N} u\left(\succsim_{i}, x\right),
$$

which is the plain utilitarian VNM SCC. The other conditions obviously hold. 


\begin{tabular}{lllllll}
\hline & $u\left(R_{1}, \cdot\right)$ & $u\left(R_{2}, \cdot\right)$ & $u\left(R_{3}, \cdot\right)$ & $u\left(R_{4}, \cdot\right)$ & $u\left(R_{5}, \cdot\right)$ & $u\left(R_{6}, \cdot\right)$ \\
\hline$x$ & 0.8 & 0 & 0.9 & 0.5 & 0.9 & 0.3 \\
$y$ & 0.9 & 1 & 1 & 0 & 0.4 & 1 \\
$z$ & 0 & 0.9 & 0.6 & 0.9 & 1 & 0 \\
$w$ & 1 & 0.7 & 0 & 1 & 0 & 0.9 \\
\hline
\end{tabular}

Table 1 Normalized VNM representations of $R_{1}, \ldots, R_{6}$

Example 3 (Strict Pareto) Let $u: V \times X \rightarrow \mathbb{R}$ be such that, for all $R \in V$, $u(R, \cdot)$ is a VNM utility function representing $R$ and $u$ is neutral. Define

$$
f\left(\left(\succsim_{i}\right)_{i \in N}\right)=\underset{x \in X}{\operatorname{argmin}} \sum_{i \in N} u\left(\succsim_{i}, x\right) .
$$

Example 4 (Separability) Consider the profile $\left(\succsim_{i}\right)_{i \in N}$. Let $\left(N_{1}, \ldots, N_{q}\right)$ be a partition of $N$ such that, for all $j, l \in\{1, \ldots, q\}$, for all $i \in N_{j}, k \in N_{l}$,

- if $j=l$, then $\exists \sigma \in \Sigma: \sigma\left(\succsim_{i}\right)=\succsim_{k}$

- if $j \neq l$, then $\nexists \sigma \in \Sigma: \sigma\left(\succsim_{i}\right)=\succsim_{k}$.

In other words, $\left(N_{1}, \ldots, N_{q}\right)$ is a partition of $N$ according to the orbits of $\mathcal{R}$. Define a new profile $\succsim_{*}$ of weak orders on $X$ where, for each $j \in\{1, \ldots, q\}$, $\# N_{j}$ voters have identical preferences, induced by $\sum_{i \in N_{j}} u\left(\succsim_{i}, \cdot\right)$, where $u(R, \cdot)$ is a normalized VNM utility representation of $R$. Define then $f\left(\left(\succsim_{i}\right)_{i \in N}\right)$ as the Copeland Social Choice Correspondence applied to the new profile ${ }_{*}$.

This VNM SCC clearly satisfies Neutrality, Anonymity and Strict Pareto. Since the statement of the VNM Comparability condition involves two preference relations belonging to a single orbit, it is easy to see that VNM Comparability is satisfied. For the same reason and because $u$ is normalized, Ordinal Comparability also holds.

Let us show that $f$ violates Separability. Suppose $X=\{x, y, z, w\}, R_{1}, \ldots, R_{6} \in$ $V$ and the normalized VNM representations of $R_{1}, \ldots, R_{6}$ are as in Table 1 . Notice that all relations $R_{1}, \ldots, R_{6}$ belong to different orbits. Let $N=\{1, \ldots, 6\}$ and $\succsim_{=}\left(\succsim_{i}\right)_{i \in N}$ be defined by $\succsim_{1}=\succsim_{2}=\succsim_{3}=\succsim_{4}=R_{1}$, $\succsim_{5}=R_{2}$ and $\succsim_{6}=R_{3}$. The corresponding profile $\succsim_{*}$ has 6 weak orders: 4 voters with preferences $w y x z, 1$ voter with $y z w x$ and 1 voter with $y x z w$. The largest Copeland score in this new profile $\succsim_{*}$ is 3 and corresponds to $w$. Hence $f\left(\left(\succsim_{i}\right)_{i \in N}\right)=\{w\}$.

Let $M=\{11, \ldots, 24\}$ and $\succsim^{\prime}=\left(\succsim_{i}^{\prime \prime}\right)_{i \in M}$ be defined by $\succsim_{11}^{\prime}=\succsim_{12}^{\prime}=\succsim_{13}^{\prime}=\succsim_{14}^{\prime}=\succsim_{15}^{\prime}=$ $R_{4}, \succsim_{16}^{\prime}=\succsim_{17}^{\prime}=\succsim_{18}^{\prime}=\succsim_{19}^{\prime}=R_{5}$ and $\succsim_{20}^{\prime}=\succsim_{21}^{\prime}=\succsim_{22}^{\prime}=\succsim_{23}^{\prime}=\succsim_{24}^{\prime}=R_{6}$. The corresponding profile $\succsim^{\prime}$ has 14 weak orders: 5 voters with preferences wzxy, 4 voters with $z x y w$ and 5 voters with $y w x z$. The largest Copeland score in this new profile $\succsim_{*}^{\prime}$ is 1 and corresponds to both $w$ and $z$. Hence $f\left(\left(\succsim_{i}^{\prime}\right)_{i \in N}\right)=\{w, z\}$.

Let us now consider the profile $\succsim^{\prime \prime}=\succsim \circ \succsim^{\prime}$. The corresponding profile $\succsim^{\prime \prime}$ has 20 voters and simple arithmetic shows that $f\left(\succsim^{\prime \prime}\right)=\{y\}$ while Separability implies $f\left(\succsim^{\prime \prime}\right)=\{w\}$.

We now prove $f$ satisfies Archimedeanness. Suppose the partition corresponding to $\succsim^{1}$ has $q$ components. The corresponding profile $\succsim_{*}^{1}$ has, for each $i \in\{1, \ldots, q\}, \# N_{i}^{1}$ identical weak orders, induced by the sum of the utilities 
in $N_{i}^{1}$. If the partition corresponding to $\succsim^{\prime}$ has $q^{\prime}$ components, then the partition corresponding to $\succsim^{1} \circ \ldots \circ \succsim^{k} \circ \succsim^{\prime}$ has then $q+s$ components, with $0 \leq s \leq q^{\prime}$. When $k \rightarrow \infty$, the corresponding profile $\left(\succsim^{1} \circ \ldots \circ \succsim^{k} \circ \succsim^{\prime}\right)_{*}$ has, for each $i \in\{1, \ldots, q\}$, at least $k \# N_{i}^{1}$ identical weak orders (the same ones as in $\succsim_{*}^{1}$ ) and, for each $i \in\{q+1, \ldots, s\}$, exactly $\# N_{i}^{\prime}$ identical weak orders, induced by the sum of the utilities in $N_{i}^{\prime}$. So, when $k \rightarrow \infty$, the Copeland rule applied to $\left(\succsim^{1} \circ \ldots \circ \succsim^{k} \circ \succsim^{\prime}\right)$ * yields a subset of $f\left(\succsim^{1}\right)$.

Example 5 (Archimedeanness) Let $u: V \times X \rightarrow \mathbb{R}$ be such that, for all $R \in V, u(R, \cdot)$ is a normalized utility function (not necessarily VNM) representing $R$ and $u$ is neutral. Define

$$
\begin{gathered}
h\left(\left(\succsim_{i}\right)_{i \in N}\right)=\underset{x \in X}{\operatorname{argmax}} \sum_{i \in N} u\left(\succsim_{i}, x\right) . \\
f\left(\left(\succsim_{i}\right)_{i \in N}\right)=\underset{x \in h\left(\left(\succsim_{i}\right)_{i \in N}\right)}{\operatorname{argmax}} \#\left\{i \in N: \bar{x} \succsim_{i} \bar{z} \text { for all } z \in X\right\} .
\end{gathered}
$$

Put differently, this VNM SCC successively applies the argmax to two different criteria: first the sum of the utilities and, then, a criterion based on the number of times an alternative is maximal in individual preferences. This VNM SCC clearly satisfies Neutrality, Anonymity and Strict Pareto.

If an alternative $x$ is selected in $f(\succsim)$, it is maximal in $\succsim$ according to the utilitarian criterion and according to the second criterion. If the same alternative $x$ is selected in $f\left(\succsim^{\prime}\right)$, it is also maximal in $\succsim^{\prime}$ according to both criteria. Since both criteria are additive, $x$ is again maximal in $\succsim 0 \succsim^{\prime}$ according to both criteria and, hence, Separability holds.

To see that $f$ satisfies Ordinal Comparability, notice that, for all profiles as in the statement of these conditions, we have

$$
\#\left\{i \in N: \bar{x} \succsim_{i} \bar{z} \text { for all } z \in X\right\}=\#\left\{i \in N: \bar{y} \succsim_{i} \bar{z} \text { for all } z \in X\right\}
$$

so that Ordinal Comparability obviously holds.

Suppose $X=\{x, y, z\}$, the normalized representation of $R$ is $u(R, x)=$ $1, u(R, y)=u(R, z)=0$ and the normalized representation of $R^{\prime}$ is $u(R, y)=$ $1, u(R, x)=0.5$ and $u(R, z)=0$. Let $N^{1}=\{2,3,4\}, M=\{1\}, \succsim^{1}=\left(\succsim_{i}^{1}\right.$ )$_{i \in N^{1}}=\left(R, R^{\prime}, R^{\prime}\right)$ and $\succsim^{\prime}=\left(\succsim_{i}\right)_{i \in M}=(R)$. Then $h\left(\succsim^{1}\right)=\{x, y\}$ and $f\left(\succsim^{1}\right.$ )$=\{y\}$. For any $k>0, h\left(\succsim^{1} \circ \ldots \circ \succsim^{k} \circ \succsim^{\prime}\right)=\{x\}$ and $f\left(\succsim^{1} \circ \ldots \circ \succsim^{k} \circ \succsim^{\prime}\right.$ )$=\{x\}$, thereby violating Archimedeanness.

We finally show that this example also violates VNM-Comparability. Let $X=\{x, y, z\}$. Suppose $u(R, \cdot)$ is a VNM utility function for all $R \in V$, $u(R, x)=1, u(R, y)=0.5, u(R, z)=0$ and $u(R, y)=1, u(R, z)=0.5$ and $u(R, x)=0$. Let $N=\{1,2\}, M=\{3\}, \succsim=\left((R)_{i \in N} \circ\left(R^{\prime}\right)_{i \in M}\right)$. Then $h(\succsim)=$ $\{x, y\}$ and $f(\succsim)=\{x\}$, thereby violating VNM-Comparability.

Example 6 (Ordinal Comparability) Let $u: V \times X \rightarrow \mathbb{R}$ be such that, for all $R \in V, u(R, \cdot)$ is a VNM utility function representing $R$ and $u$ is neutral but not normalized. Define

$$
f\left(\left(\succsim_{i}\right)_{i \in N}\right)=\underset{x \in X}{\operatorname{argmax}} \sum_{i \in N} u\left(\succsim_{i}, x\right) .
$$


This VNM SCC clearly violates Ordinal Comparability and satisfies all other conditions.

Example 7 (VNM-Comparability) Let $g: V \times X \rightarrow \mathbb{R}$ be such that, for all $R \in V, g(R, \cdot)$ is a VNM utility function representing $R$, with $g$ neutral. Define $u: V \times X \rightarrow \mathbb{R}$ by $u(R, x)=(g(R, x))^{3}$ for all $R \in V$ and $x \in X$. Define

$$
f\left(\left(\succsim_{i}\right)_{i \in N}\right)=\underset{x \in X}{\operatorname{argmax}} \sum_{i \in N} u\left(\succsim_{i}, x\right) .
$$

This VNM SCC violates VNM-Comparability because $u(R, \cdot)$ is the third power of a VNM utility function representing $R$. It is therefore monotonically but not linearly related to a VNM utility function representing $R$. It clearly satisfies all other conditions.

\section{Discussion}

\subsection{The choice of $u$}

Suppose a social planner buys all axioms of Theorem 1 and therefore wants to use an anonymous utilitarian VNM SCC. Yet, she does not adhere to Ordinal Comparability and, hence, she does not want to use the relative utilitarian VNM SCC. She then faces a choice: among the infinite family of anonymous utilitarian VNM SCCs, which one is she going to use? Is there a reasoned way to select a specific member of this family? Our results do not answer this question, but Theorem 2 shows a possible direction: instead of imposing Ordinal Comparability in terms of maximal and minimal elements, we could enrich our primitives with two particular alternatives with an identical meaning to all voters (called interpersonally significant norm by Blackorby and Donaldson [1982]), and restate Ordinal Comparability in terms of these two particular alternatives. The existence and meaningfulness of such interpersonally significant norms is another debate. Other directions are perhaps possible.

\section{$5.2 \mathrm{SCC}$ vs SWF}

Our results are stated in terms of a social choice correspondence while most of the literature about cardinal social choice is stated in terms of social welfare function $(\mathrm{SWF})$. In particular, the characterization of relative utilitarianism by Dhillon and Mertens [1999] is in terms of a SWF.

It is easy to restate our Theorem 1 in terms of SWFs. We just need to restate the VNM-Comparability condition.

A 10 VNM-Comparability $(S W F)$. There exists an infinite subset $O$ of $\mathbb{N}$ such that, whenever

$-R \in V$ is such that $\bar{x} P \bar{y} P \bar{z}$ and $\bar{y} I \lambda \bar{x}+(1-\lambda) \bar{z}$, 
- $N^{1}$ and $N^{2}$ are disjoint subsets of $O$,

$-\sigma \in \Sigma$ is such that $\sigma(x)=y, \sigma(z)=x$

$-\# N^{2} / \# N^{1}=1-\lambda$,

then $x$ and $y$ are indifferent in the social preferences corresponding to profile $(R)_{i \in N^{1}} \circ(\sigma(R))_{i \in N^{2}}$.

We then have the following conjecture, using the new VNM-Comparability plus the conditions of Smith [1973] and a Pareto condition.

Conjecture 1 Let $\# X \geq 3$. A VNM SWF satisfies Neutrality, Anonymity, Separability, Archimedeanness, Weak Pareto and VNM-Comparability iff it is the anonymous utilitarian VNM SWF, that is, $x$ is socially weakly preferred to $y$ in the profile $\left(\succsim_{i}\right)_{i \in N}$ iff $\sum_{i \in N} u\left(\succsim_{i}, x\right) \geq \sum_{i \in N} u\left(\succsim_{i}, y\right)$.

Proving this conjecture would involve proving first a variant of Proposition A1 in [Pivato, 2014], recasted in terms of a SWF. This is beyond the scope of this paper.

\subsection{Other measurement techniques}

In this paper, in order to obtain cardinal preferential information about the finite set of alternatives, we embed them in a rich set (the set of all lotteries) and we observe the preferences of the voters on this rich set. Provided the preferences satisfy some properties, it is possible to infer some cardinal preferential information about the alternatives.

Notice that there are other ways to obtain cardinal preferential information about a finite set of alternatives. Suppose the alternatives are elements of a Cartesian product. For instance $X=X_{1} \times X_{2}$ where $X_{1}=\{100,110,120\}$ and $X_{2}=\{30,40,50\}$ are amounts to be invested in two different projects. We can embed the set $X$ in the richer set $[100,120] \times[30,50]$ and observe the preferences of the voters over this richer set. Provided their preferences satisfy some conditions, using techniques of conjoint measurement, it is possible to represent the preferences by means of two utility functions unique up to positive affine transformations [Debreu, 1960, Krantz et al., 1971]. Restating Proposition 1 for such preference relations is immediate. It is then probably not too difficult to devise a new comparability condition, akin to VNM Comparability, in order to characterize anonymous utilitarianism in this context. Techniques of algebraic difference measurement or extensive measurement can also be used [Krantz et al., 1971]. In the latter case, the utilities are unique up to a positive linear transformation (ratio scale) and this may lead to a subset of the anonymous utilitarian family.

An interesting consequence of our approach (without individual utilities) is that the exact form of the comparability condition would depend on the measurement technique (decision under risk, conjoint measurement, etc.) while, with the classical approach (social welfare functional acting on profiles of individual utilities), the same invariance condition is used irrespective of what utilities mean and of the way they have been measured. 
Acknowledgements I am grateful to Antoinette Baujard, Denis Bouyssou, Marc Pirlot, John Weymark and two anonymous reviewers for comments and discussions.

\section{References}

K. J. Arrow. Social choice and individual values. Wiley, New York, 2nd edition, 1963.

C. Blackorby and D. Donaldson. Ratio-scale and translation-scale full interpersonal comparability without domain restrictions: Admissible socialevaluation functions. International Economic Review, 23(2):249-268, 1982.

T. Börgers and Y. M. Choo. A counterexample to Dhillon (1998). Social Choice and Welfare, 48(4):837-843, 2017a.

T. Börgers and Y. M. Choo. Revealed relative utilitarianism. Unpublished, 2017b.

W. Bossert. On intra- and interpersonal utility comparisons. Social Choice and Welfare, 8:207-219, 1991.

C. P. Chambers and F. Echenique. When does aggregation reduce risk aversion? Games and Economic Behavior, 76:582 - 595, 2012.

C. d'Aspremont and L. Gevers. Equity and the informational basis of collective choice. Review of Economic Studies, 44:199-209, 1977.

G. Debreu. Topological methods in cardinal utility theory. In K. J. Arrow, S. Karlin, and P. Suppes, editors, Mathematical Methods in the Social Sciences, pages 16-26. Stanford University Press, Stanford, 1960.

A. Dhillon. Extended Pareto rules and relative utilitarianism. Social Choice and Welfare, 15(4):521-542, 1998.

A. Dhillon and J. F. Mertens. Relative utilitarianism. Econometrica, 67(3): 471-498, 1999.

D. Donaldson and J. A. Weymark. Social choice in economic environments. Journal of Economic Theory, 46:291-308, 1988.

M. Fleurbaey and S. Zuber. Fair management of social risk. Journal of Economic Theory, 169:666-706, 2017.

J. C. Harsanyi. Cardinal welfare, individual ethics, and interpersonal comparisons of utility. Journal of Political Economy, 63(4):309-321, 1955.

N. E. Jensen. An introduction to Bernouillian utility theory I: utility functions. Swedish Journal of Economics, 69:163-183, 1967.

D. H. Krantz, R. D. Luce, P. Suppes, and A. Tversky. Foundations of Measurement: Additive and Polynomial Representations. Academic Press, New York, 1971.

T. Marchant. Scale invariance and similar invariance conditions for bankruptcy problems. Social Choice and Welfare, 31:693-707, 2008.

M. Morreau and J. A. Weymark. Measurement scales and welfarist social choice. Journal of Mathematical Psychology, 75:127-136, 2016.

R. B. Myerson. Axiomatic derivation of scoring rules without the ordering assumption. Social Choice and Welfare, 12:59-74, 1995. 
M. Pivato. Formal utilitarianism and range voting. Mathematical Social Sciences, 67:50-56, 2014.

K. W. S. Roberts. Interpersonal comparability and social choice theory. Review of Economic Studies, 47:421-439, 1980.

J. E. Roemer. Theories of distributive justice. Harvard University Press, 1996.

A. K. Sen. Collective choice and social welfare. Holden-Day, San Francisco, 1970 .

A. K. Sen. Welfare inequalities and Rawlsian axiomatics. Theory and Decision, 7:243-262, 1976 .

J. H. Smith. Aggregation of preferences with variable electorate. Econometrica, 41:1027-1040, 1973.

Y. Sprumont. On relative egalitarianism. Social Choice and Welfare, 40(4): 1015-1032, 2013.

W. Thomson. On the axiomatic method and its recent applications to game theory and resource allocation. Social Choice and Welfare, 18:327-386, 2001.

J. A. Weymark. A reconsideration of the Harsanyi-Sen debate on utilitarianism. In J. Elster and J. E. Roemer, editors, Interpersonal Comparisons of Well-Being. Cambridge University Press, Cambridge, 1991.

H. P. Young. Social choice scoring functions. SIAM Journal on Applied Mathematics, 28:824-838, 1975. 